\title{
Geomorphological innovation through advances in geovisualisation
}

\author{
Antoni B Moore ${ }^{\mathrm{a}, *}$, Mike J Smith ${ }^{\mathrm{b}}$ \\ a School of Surveying, University of Otago, New Zealand, tony.moore@otago.ac.nz \\ ${ }^{b}$ School of Geography and Environmental Sciences, Ulster University, Northern Ireland, editor@journalofmaps.com \\ * Corresponding author
}

Keywords: geomorphology, mixed reality, visual analytics, art, geovisualisation

\begin{abstract}
:
Geomorphology (the study of Earth surface landforms) is a discipline that requires effective geovisualisation in four dimensions; that is landforms in 3D space, and their change through time. For example, in alpine environments, the 3D shapes of landforms are subject to processes of glacial erosion and deposition that cause them, over time, to be created, evolve and eventually, be destroyed. Until recently, the principal visualisation in this domain was through geomorphological mapping in 2D and 3D (Smith et al, 2013).
\end{abstract}

Advances in science, technology and art have changed the "landscape" of geovisualisation in geomorphology, predominantly through mixed reality environments, visual exploration (e.g. of "Big Data" through the collation of vast environmental datasets) and approaches that are complementary with the increasing global human impact. These, in turn, afford opportunities for future research and development, the topic of this submission.

Recent changes, particularly technology-led, have led geovisualisation to embrace 3D and 4D approaches. Examples include virtual reality, augmented reality, innovative static space-time cube displays and the efficient handling of dynamic visualisation (animation). However, it could be argued there have been missed opportunities in visualisation in the past. Geomorphological mapping activities were and are exclusively visual, which makes them ripe for experimentation with non-visual channels such as sound, but particularly with tactile and haptic communication in the study of landforms, where shape, (textural) structure and volume are particularly important.

In the case of VR, there is the acknowledged facilitation of indirect interaction with, and representation of, hazardous and extraterrestrial locations, as well as past and future time points. The future potential of VR in particular can be seen to include exploration of the property of embodiment (e.g. along the lines of Ahn et al, 2016, for coral bleaching), where users could be induced to 'feel' geomorphological process through first-person landform avatars (e.g. a retreating glacier).

Through mixed reality as a whole, there is now an ambiguity with where visualisation takes place in relation to the natural geomorphological environment. While VR tries to replicate the environment in the lab, AR allows seamless visualisation in situ. AR, and in particular tangible AR instances such as sandtables, also gives direct control of physical 'toy' environments allowing for ease of experimentation (e.g. Moore et al, 2020). This has been a real advance for physical geographic education (e.g. Virtual Field Trips) and research.

Fundamentally, the role of the human is inescapable in visualisation, which now has a central function of dynamic interaction as well as representation / mapping. Therefore, human-led aspects of visualisation such as cognition, usability testing and personalised maps and interfaces (through programming) are as a result open to geomorphology. The creation and adaptation of games for geomorphological education and research represents an opportunity (partially realised in the urban geographic domain) as a geovisualisation means already familiar to a large group of potential users.

The natural environment now has the indelible imprint of humans, which has lent added meaning to the application of art to geomorphology. There is an increasing recognition that art can provide a humanistic alternative to scientific capture, analysis and visualisation (Tooth et al, 2019), with geomorphologists and artists collaborating on artworks that represent a world demonstrating a dominant anthropogenic signature. 
Geomorphologists have had limited impact in artistic renditions of landscape in the past. This is also true of landscape spatialisations (using landscape in a cognitively plausible manner to represent non-spatial data), which should communicate through our shared geomorphological knowledge (Fabrikant et al, 2010). Geomorphologists can also contribute their expertise in landform and process to virtual and game environments too. These, along with developments associated with immersive environments themselves (e.g. embodiment) and visual exploration (e.g. of Big Data), are areas of opportunity for future geomorphological geovisualisation. 